\title{
IL PROBLEMA COSTITUZIONALE DELLATTUAZIONE DEI DIRITTI SOCIALI
}

THE CONSTITUTIONAL PROBLEM OF THE IMPLEMENTATION OF SOCIAL RIGHTS 


\title{
IL PROBLEMA COSTITUZIONALE DELL'ATTUAZIONE DEI DIRITTI SOCIALI
}

\author{
THE CONSTITUTIONAL PROBLEM OF THE \\ IMPLEMENTATION OF SOCIAL RIGHTS
}

\begin{abstract}
Riassunto: L'articolo si interroga sul problema dell'attuazione dei diritti costituzionali, e in particolare dei diritti sociali, nel quadro di una costituzione pluralista. Considerando i diritti costituzionali vincoli all'esercizio dei pubblici poteri - in quanto contenuto del patto costituente, prima ancora che per via della loro collocazione gerarchica -, si argomenta che, pur nell'ambito della loro discrezionalità politica, i pubblici poteri stessi hanno il dovere di realizzare interventi volti all'attuazione dei diritti (e di astenersi dal compiere interventi ostativi alla loro attuazione). Ciò vale anche per la decisione di bilancio, che deve rispettare le priorità costituzionali, privilegiando le «spese costituzionalmente vincolate» rispetto alle «spese costituzionalmente facoltative» (ed evitare le «spese costituzionalmente vietate»). In vista del raggiungimento di questo obiettivo, si suggerisce la configurazione di vincoli, costituzionalmente giustiziabili, non $d i$ ma al bilancio, vale a dire in favore della destinazione di risorse certe agli interventi pubblici volti a dare concretezza al contenuto minimo dei diritti costituzionali.
\end{abstract}

Parole-chiavi: attuazione dei diritti costituzionali, diritti sociali, bilancio

\begin{abstract}
The article deals with the problem of the implementation of constitutional rights, particularly of constitutional social rights, within the framework of a pluralist constitution. Considering the constitutional rights constraints on the exercise of public powers - as a content of the constitutional pact more than due to their hierarchical position -, it is argued that, even within their political discretion, the public powers themselves have a duty to carry out interventions aimed at the implementation of rights (and to refrain from carrying out interventions that hinder their implementation). This also applies to the budgetary decision, which must respect constitutional priorities, favoring "constitutionally bound expenditure" with respect to "constitutionally voluntary expenses" (and avoiding "constitutionally prohibited expenses"). In view of the achievement of this goal, we suggest the configuration of
\end{abstract}


constraints, constitutionally justiciable, to budget in favor of the destination of certain resources to public interventions aimed at giving substance to the minimum content of constitutional rights.

Keywords: implementation of constitutional rights, social rights, budget

\section{1. - I diritti sociali nel quadro di una costituzione pluralista}

In questo articolo mi propongo di svolgere alcune riflessioni sul tema dell'attuazione dei diritti costituzionali - e, in particolare, dei diritti sociali - nell'ambito dell'esperienza costituzionale italiana, anche se con l'obiettivo di formulare considerazioni valevoli, in generale, per le costituzioni pluraliste.

\section{1. - Pluralismo e bilanciamento}

Il pluralismo è caratteristica che accomuna le costituzioni del Novecento, come effetto del superamento del voto per censo e del conseguente allargamento dei confini della cittadinanza politica a tutte le classi sociali: quell'allargamento che - per ricorrere al linguaggio di Massimo Severo Giannini - nella prima metà del secolo trasforma lo Stato da monoclasse a pluriclasse. In uno scenario in cui gli attori politici diventano molteplici ed esprimono visioni del mondo diverse - se non, su taluni profili, contrapposte - le costituzioni diventano documenti riassuntivi delle variegate tendenze culturali, economiche e politiche che è necessario far convivere nella società.

In questa prospettiva, la contestuale presenza nella costituzione di una pluralità di princìpi - anche tra loro contrastanti e comunque non gerarchizzati - è un dato positivo, perché tesse, anziché lacerare, il legame sociale. Includere il più ampio numero di visioni in competizione, anziché escluderne a forza alcune, consente alle carte fondamentali di operare come strumenti di convivenza tra forze sociali contrapposte, sostituendo alla logica dello scontro fratricida amiconemico (à la Carl Schmitt) quella del conflitto tra avversari politici (secondo la teorizzazione di Hans Kelsen). Sul piano politico, il conflitto riguardo al contenuto da dare alla costituzione si trasforma in conflitto sull'attuazione di quel contenuto, vale a dire sulla scelta, rimessa alla discrezionalità politica, dell'ordine e dell'intensità di realizzazione concreta delle diverse previsioni costituzionali, pur con l'onere di non dimenticarne nessuna. Sul piano giuridico, è il bilanciamento lo strumento attraverso cui princìi diversi trovano, contestualmente, parziale attuazione nella regola di volta in volta sancita dal legislatore o dal giudice.

Nell'ottica del bilanciamento, i princìpi sono destinati a limitarsi reciprocamente, dando vita a un sistema costituzionale sempre aperto al possibile raggiungimento di nuovi punti di equilibrio, rappresentati dalle regole nel tempo concretamente stabilite. Il compito 
di scegliere tra i vari possibili punti di equilibrio spetta al legislatore: le scelte effettuabili sono molteplici e quella che prevarrà dipende dai rapporti di forza, sempre mutevoli, in un dato momento vigenti nella società. Vi è, tuttavia, un limite invalicabile, che vincola la discrezionalità legislativa (anch'essa inevitabilmente limitata, come tutti i poteri costituiti): il sacrifico del principio che in un determinato frangente è socialmente e politicamente meno "sostenuto" non potrà mai spingersi sino al suo completo annullamento, pena il prodursi di uno "sbilanciamento" incostituzionale sanzionabile dal giudice delle leggi. È ciò che Luigi Ferrajoli - pur critico nei riguardi della nozione di bilanciamento - definisce la «sfera del non decidibile».

La Corte costituzionale italiana si è espressa in argomento con grande chiarezza, attraverso le sentenze n. 264 del 2012 e n. 85 del 2013. La sentenza n. 264 del 2012 afferma che compito della Corte costituzionale è proteggere la Costituzione come un insieme unitario, in modo da assicurare una tutela «sistemica e non frazionata» di tutti i diritti e i princìpi in essa presenti. Quel che, in particolare, non deve accadere - ha aggiunto la sentenza n. 85 del 2013 - è «l'illimitata espansione di uno dei princìpi, che diverrebbe "tiranno" nei confronti delle altre situazioni giuridiche costituzionalmente riconosciute e protette», ed è per questo che la «Costituzione italiana, come le altre Costituzioni democratiche e pluraliste contemporanee, richiede un continuo e vicendevole bilanciamento tra princìpi e diritti fondamentali, senza pretese di assolutezza per nessuno di essi».

Si potrebbe dire così: la tecnica del bilanciamento prevede che ogni principio vada rispettato nella sua intima essenza, perché non farlo significherebbe espungerlo dal compromesso costituzionale che ha dato unità al pluralismo. La scienza giuridica ha parlato, in proposito, di «nocciolo duro», «contenuto minimo», «livello essenziale», «soglia invalicabile», «elemento incomprimibile», «nucleo indefettibile» ecc. dei princìpi costituzionali. Non è senza rilievo che tale nozione abbia trovato positivizzazione nell'ordinamento costituzionale italiano, nel 2001, con la riscrittura dell'art. 117, comma 2, lett. m, Cost. per il quale i «livelli essenziali delle prestazioni concernenti i diritti civili e sociali [...] devono essere garantiti su tutto il territorio nazionale». Ciò comporta - ha precisato la Corte costituzionale - che «nella individuazione delle misure necessarie a tutela dei diritti delle persone», «il legislatore [...] gode di discrezionalità», ma «detto potere discrezionale non ha carattere assoluto e trova un limite nel rispetto di un nucleo indefettibile di garanzie per gli interessati» (sentenza n. 80 del 2010).

Ciò detto, un elemento di potenziale perplessità merita di essere sottolineato: una volta positivizzata, la nozione di «livello essenziale» - configurabile come limite di costituzionalità "in negativo" alla legislazione, finché frutto di teorizzazione dottrinale - 
diventa per il legislatore obiettivo da realizzare "in positivo", con il rischio evidente che quello che dovrebbe essere il livello minimo di tutela dei diritti diventi il livello di tutela dei diritti, oltre il quale si perde ragione di andare. Per ricorrere a una metafora: che ciò che nella costruzione dell" "edificio" dei diritti dovrebbe essere il "pavimento" si trasformi nel "tetto".

\section{2. - E l'equilibrio di bilancio?}

Il discorso si fa ulteriormente delicato in seguito all'inserimento nella Costituzione italiana del principio dell'equilibrio di bilancio (1. cost. 20 aprile 2012, n. 1). Con riferimento al discorso in svolgimento, il tema, ricco di implicazioni, rileva soprattutto per una questione: il principio dell'equilibrio dei bilanci (statale, regionali, locali) è un super-principio costituzionale «imperioso»o «tiranno», destinato a prevalere in ogni caso sugli altri princìpi, o è un principio costituzionale posto sullo stesso piano degli altri princìpi costituzionali e dunque, al pari degli altri, suscettibile di bilanciamento?

Sulla base della ricostruzione sopra prospettata a proposito del pluralismo costituzionale, non si può che affermare la necessaria ponderabilità anche dell'equilibrio di bilancio: tutti i princìpi costituzionali, nessuno escluso, devono essere bilanciabili, a pena di far cadere l'intero pluralismo costituzionale. Se anche un solo principio risultasse non bilanciabile, se ne dovrebbe infatti dedurre che quel principio vale in assoluto: ne deriverebbe l'assolutismo, non il pluralismo, costituzionale. Senza considerare che ritenere l'equilibrio di bilancio un superprincipio non ponderabile causerebbe, come inaccettabile conseguenza, l'inversione dell'ordine gerarchico delle fonti, con la subordinazione della Costituzione alla legislazione (di bilancio) ordinaria: i diritti risulterebbero infatti attuabili solo se, e nella misura in cui, fossero loro destinate risorse dalla legge di bilancio.

A conforto di tale ricostruzione - per la quale la ponderazione deve necessariamente interessare tutti i princìpi costituzionali, nessuno escluso - si può richiamare la prima sentenza pronunciata dalla Corte costituzionale, la n. 1 del 1956, adottata in polemica con la Corte di cassazione proprio per via della differente concezione dei diritti costituzionali fatta valere dalle due supreme magistrature. Ridotta all'essenziale, la questione verteva intorno al tentativo - sostenuto dalla Cassazione - di vincolare la normatività delle disposizioni costituzionali di programma (in particolare quelle che prevedono la realizzazione di uno Stato sociale) alla previa adozione della legislazione ordinaria di attuazione (Sezioni Penali Unite, sentenza del 7 febbraio 1948). Di contro, la Consulta approfittò proprio della prima occasione in cui poté pronunciarsi per tagliare il nodo che si voleva stringere al collo della Costituzione, proclamando la prescrittività di tutte le disposizioni costituzionali, incluse quelle di programma e di principio.

In definitiva, allora come oggi, il riconoscimento costituzionale di un diritto non è 
un atto meramente formale, la cui attuazione normativa è rimessa alla buona volontà del legislatore; al contrario, produce un vero e proprio vincolo giuridico. Introdurre un diritto in una carta costituzionale significa porre un vincolo alla discrezionalità politica, che da quel momento deve predisporre gli apparati necessari a far fronte alle esigenze riconducibili a quel diritto. Naturalmente, occorre considerare anche gli altri interessi costituzionali coinvolti tra cui, oggi in molti Paesi, l'esigenza che i bilanci pubblici siano «in equilibrio» - e, come visto, bilanciarli: com'è accaduto, con esiti diversi, nelle sentenze della Corte costituzionale italiana n. 264 del 2012, n. 10, n. 70, n. 178 del 2015 e, da ultimo, n. 275 del 2016.

\section{2. - Crisi e diritti sociali}

Il discorso sin qui svolto vale, a maggior ragione, in tempi di crisi economica, quando la contrazione delle risorse disponibili tende, anzitutto, a ripercuotersi sulle prestazioni che costituiscono il sistema di welfare pubblico.

I dati relativi alla condizione economico-sociale italiana sono a tratti drammatici, nonostante - occorre ricordarlo - l'Italia continui a essere una delle dieci economie più floride del pianeta. Il problema fondamentale, in effetti, non è tanto quello della carenza di risorse, quanto, piuttosto, quello della loro sempre più diseguale distribuzione, al punto che l'Ocse colloca l'Italia nel gruppo dei Paesi in cui, nei dieci anni della crisi, la disuguaglianza sociale, provocata dalla concentrazione delle risorse economiche (reddito e patrimonio) verso l'alto, è maggiormente aumentata. E così, mentre l'Eurostat stima che l'Italia conti, in termini assoluti, il più alto numero di poveri in Europa (10,4 milioni su un totale 78,5), 1'associazione italiana dei gestori del risparmio (Assogestioni) dichiara che negli ultimi dieci anni l'ammontare delle risorse affidate a fondi d'investimento e gestioni di portafogli è sempre aumentato, sino a toccare la cifra record di 2.089miliardi di euro nel 2017 (a fronte degli 841,2 miliardi del 2008). L'indice di Gini certifica chiaramente questa situazione, collocando l'Italia al settimo posto in Europa per tasso di diseguaglianza, ben oltre la media dell'Unione europea.

A incidere sulla diseguaglianza sono soprattutto due elementi.

Il primo è l'inadeguatezza del sistema tributario, che - a tacere dell'abnorme evasione - ha da tempo abbandonato la strutturazione progressiva, che costituzionalmente dovrebbe essergli propria, per assumerne una sostanzialmente proporzionale a vantaggio dei più ricchi. È un discorso che vale per l'imposizione sui redditi, così come per quella sui patrimoni (con riguardo a questi ultimi, la situazione è così anomala da aver indotto l'Ocse e il Fmi a suggerire l'introduzione di un'imposta patrimoniale e un incremento delle imposte sulle successioni). Straniante, sul punto, l'andamento del dibattito pubblico contemporaneo, completamente assorbito da proposte - quali la flat tax e il "recupero"dei c.d. residui fiscali 
da parte delle regioni più ricche - che ulteriormente allargherebbero la forbice tra indigenti e benestanti, anziché da iniziative - quali la lotta all'evasione, l'ampliamento della base imponibile, la ristrutturazione secondo progressività dell'imposizione su redditi e patrimoni - rivolte al contrasto e alla riduzione delle disparità.

Il secondo elemento all'origine della diseguaglianza è la vertiginosa contrazione della spesa sociale, da cui derivano crescenti difficoltà d'intervento a favore dei più deboli, quantomeno per lenire ex post le conseguenze della povertà. Tutti i principali diritti sociali risultano in stato di grave sofferenza: la salute, l'assistenza, l'istruzione, l'abitazione. Per non dire del lavoro. Non è questa la sede in cui si può approfondire l'argomento. Ai fini del discorso in atto, è sufficiente ricordare poche cifre: $(a)$ con il 9,2\% rispetto al Pil, l'Italia spende oramai in salute meno di Regno Unito, Spagna, Francia e Germania. Gli indicatori che misurano la salute dei cittadini restano per il momento superiori alla media europea, ma ci si chiede se ancora sarà così quando - come previsto dall'ultimo Documento di economia e finanza (Def) nel 2019 il finanziamento scenderà al 6,4\% del Pil, un decimale al di sotto del livello minimo fissato dall'Ocse affinché un sistema sanitario possa adeguatamente funzionare; $(b)$ il Fondo nazionale per le politiche sociali - strumento principe di intervento in materia di assistenza - ammontava nel 2002 a 1,09 miliardi di euro; dieci anni dopo era precipitato a 42,9 milioni. Risalito sopra la soglia dei 300 milioni negli anni successivi, è ridisceso a 77 milioni nel 2017: vale a dire, ad appena il 7\% dell' ammontare di un tempo. A ciò vanno aggiunti i tagli che hanno colpito i trasferimenti statali agli enti territoriali (la Corte dei Conti ne ha certificati per 40 miliardi di euro tra il 2008 e il 2015), considerato che da tali enti dipende una parte significativa del sistema di assistenza sociale; $(c)$ la spesa in istruzione, colpita dalle draconiane decurtazioni degli anni 2008-2012 (pari ad almeno 10 miliardi di euro), colloca oramai 1'Italia agli ultimi posti in Europa, con una quota di risorse impiegate rispetto al Pil di appena il 4\% (e conseguenti dati statistici disastrosi riguardo ad abbandono scolastico, percentuale di diplomati e laureati, analfabetismo funzionale); $(d)$ la spesa in edilizia residenziale pubblica è stata praticamente azzerata, nonostante la disponibilità di abitazioni pubbliche sia oggi in grado, sul territorio nazionale, di far fronte alle esigenze abitative di 700 mila famiglie, pari ad appena un terzo di quelle che avrebbero realmente necessità di un alloggio.

\section{3. - Dai vincoli di bilancio ai vincoli al bilancio}

Nel quadro della situazione ora sommariamente descritta, il tema dell'attuazione dei diritti costituzionali diventa, a maggior ragione, ineludibile. Se già è problematico assicurare adeguata attuazione a tutti i diritti quando le circostanze economiche sono favorevoli, che fare nei contesti di crisi economica? 
Un'indicazione di direzione sembra emergere da quanto detto nei paragrafi iniziali a proposito del pluralismo costituzionale e del conseguente necessario bilanciamento tra $\mathrm{i}$ diritti. L'indicazione è la seguente: se è vero che tutti i diritti vanno, quantomeno, attuati con riguardo al loro «contenuto minimo essenziale», non tutti gli impieghi delle risorse pubbliche risultano allora equivalenti, ma occorre distinguere tra «spese costituzionalmente vincolate», «spese costituzionalmente facoltative» e «spese costituzionalmente vietate». «Spese costituzionalmente vincolate» sarebbero quelle che il legislatore deve necessariamente disporre, in quanto rivolte alla soddisfazione dei livelli essenziali delle prestazioni inerenti ai diritti civili e sociali. «Spese costituzionalmente facoltative» sarebbero quelle che, solo a fronte della eventuale disponibilità di risorse eccedenti, il legislatore è libero di decidere discrezionalmente, al fine di finanziare attività, prestazioni o servizi diversi da quelli inerenti al «nucleo duro» dei diritti. «Spese costituzionalmente vietate» sarebbero, infine, quelle volte a realizzare obiettivi il cui raggiungimento è precluso dalla costituzione (come, per fare un facile esempio relativo ai Paesi in cui è vietata la pena capitale, le spese rivolte a costruire strutture in cui dare esecuzione a sentenze di morte e ad acquistare le strumentazioni a tal fine necessarie).

Si può dire così: posta l'inammissibilità delle «spese costituzionalmente vietate», le «spese costituzionalmente vincolate» devono avere la «priorità» sulle «spese costituzionalmente facoltative», dal momento che queste ultime risultano costituzionalmente legittime solo se, una volta soddisfatte le prime, avanza disponibilità di risorse. Altrimenti, si configurerebbe un utilizzo incostituzionale della discrezionalità politica, contro il quale sarà possibile chiamare a intervenire i giudici costituzionali. Il legislatore agisce, infatti, incostituzionalmente sia se non prevede «spese costituzionalmente necessarie», sia se prevede «spese costituzionalmente facoltative» senza che prima siano state soddisfatte tutte le «spese costituzionalmente necessarie», sia, infine, se prevede «spese costituzionalmente vietate». Se così è, nei Paesi a costituzione pluralista la Corte costituzionale deve allora essere messa in condizione di controllare la manovra di bilancio decisa dal Parlamento, eventualmente colpendola con dichiarazione di incostituzionalità. Come ha scritto Gustavo Zagrebelsky, per le spese che corrispondono a veri e propri diritti di prestazione sanciti dalla Costituzione «non c'è politica (e quindi nemmeno dipendenza da interpositio di valutazioni discrezionali del legislatore) ma solo giurisdizione in nome della Costituzione».

In concreto, si possono distinguere tre ipotesi.

La prima ipotesi è quella dell'inadeguatezza dell' ammontare complessivo delle risorse pubbliche disponibili, per cui, anche se non sono state previste «spese costituzionalmente facoltative», non è comunque possibile soddisfare tutte le «spese costituzionalmente vincolate». 
In tal caso, sebbene la scelta del quantum spendere spetti al legislatore, se le condizioni economiche complessive sono tali da consentire una maggiore spesa (grazie a un maggiore prelievo fiscale), la Corte costituzionale dovrebbe poter dichiarare l'incostituzionalità della legge di bilancio per carenza di entrate. L'ipotesi è presa esplicitamente in considerazione da Massimo Luciani, che, con riguardo all'ordinamento italiano, immagina due possibili strumenti di intervento: (1) l'«adozione di una sentenza parziale, nella quale enunciare il "principio" dell'incostituzionalità della legge in assenza del reperimento delle risorse necessarie entro il termine indicato, con contestuale fissazione di una nuova udienza subito dopo la scadenza di tale termine. A seguito della seconda udienza si potrebbe adottare la sentenza definitiva, che - ovviamente - sarebbe di rigetto o di accoglimento a seconda della risposta data dal legislatore»; (2) l'adozione di «una sentenza di incostituzionalità condizionata e differita. La Corte potrebbe dichiarare la legge illegittima a condizione che il legislatore non abbia reperito, entro l'esercizio finanziario indicato, le risorse necessarie, con effetti annullatori decorrenti a far data dal maturarsi dell'inadempimento».

La seconda ipotesi è quella in cui, pur in presenza di un'adeguata raccolta di risorse attraverso il sistema fiscale, si sia in presenza di «spese costituzionalmente vietate». A prescindere dal fatto che ciò renda o meno insufficienti le risorse disponibili per le «spese costituzionalmente vincolate», la Corte costituzionale dovrebbe poter dichiarare l'incostituzionalità della legge di bilancio perché prevede decisioni di spesa contrarie alla costituzione. Come strumento utilizzabile si potrebbe pensare a sentenze di accoglimento parziale che annullano la legge di bilancio nella parte in cui prevede spese contrarie alla costituzione, con l'effetto di liberare risorse per le «spese costituzionalmente vincolate», se insufficienti, o per ulteriori «spese costituzionalmente facoltative» se le «spese costituzionalmente vincolate» sono già adeguatamente finanziate.

La terza ipotesi è quella in cui, pur in presenza di un'adeguata raccolta di risorse attraverso il sistema fiscale, vi sia un eccesso di «spese costituzionalmente facoltative», tale da rendere insufficienti le risorse disponibili per le «spese costituzionalmente vincolate». In tal caso,grazie a un giudizio di ragionevolezza, la Corte costituzionale dovrebbe poter dichiarare l'incostituzionalità della legge di bilancio per cattivo utilizzo della discrezionalità politica nella scelta d'impiego delle risorse tramite sentenze additive di principio che colpiscono la legge di bilancio nella parte in cui non consente di attribuire adeguate risorse per l'attuazione del livello minimo essenziale dei diritti costituzionali. L'ipotesi è presa in considerazione, nella dottrina italiana, da Cesare Pinelli che immagina la possibilità di impugnare innanzi alla Corte costituzionale le leggi di stabilità e di bilancio in caso di «manifesta inadeguatezza delle risorse necessarie al finanziamento dei livelli essenziali delle prestazioni relative ai 
diritti fondamentali»: operando tramite lo strumento della pronuncia additiva di principio, la Corte avrebbe modo di controllare le scelte di bilancio compiute dal legislatore senza invaderne l'ambito di competenza, dal momento che il Parlamento manterrebbe piena discrezionalità nel decidere come riequilibrare il bilancio, scegliendo quali risorse assegnate a «spese costituzionalmente facoltative» ricollocare in favore delle «spese costituzionalmente vincolate» finanziate in misura insufficiente. C'è, peraltro, almeno un precedente in cui la stessa Corte costituzionale italiana si è spinta oltre: è il caso della sentenza n. 241 del 1989 che, con riferimento al diritto all'abitazione, ha dichiarato incostituzionale l'art. 22, co. 2, della legge finanziaria n. 67 del 1988 perché aveva arbitrariamente sottratto una parte cospicua (5 mila miliardi di lire) del contributo Gescal destinato alla costruzione di abitazioni per i lavoratori dipendenti, assegnandolo al bilancio generale dello Stato.

Concludendo, la possibile soluzione capace di garantire il rispetto delle priorità costituzionali di spesa potrebbe essere quella di configurare vincoli, costituzionalmente giustiziabili, non $d i$ ma al bilancio, vale a dire in favore della destinazione di risorse certe all'attuazione del contenuto minimo dei diritti. Come qui sostenuto, è possibile ricavare tali vincoli attraverso l'interpretazione del testo costituzionale. Ma si potrebbe andare oltre: Gianni Ferrara ha proposto di modificare la Costituzione italiana inserendovi una «riserva di bilancio in favore dei diritti sociali» in modo «che nei bilanci di previsione dello Stato, delle regioni, dei comuni il cinquanta per cento della spesa risulti complessivamente destinato a garantire direttamente o anche indirettamente i diritti: alla salute, all'istruzione, alla formazione e all'elevazione professionale delle lavoratrici e dei lavoratori, alla retribuzione proporzionata alla quantità e qualità del lavoro, all'assistenza sociale, alla previdenza, all'esistenza dignitosa ai lavoratori e delle loro famiglie».

\section{Bibliografia}

A.B. Atkinson, Disuguaglianza. Cosa si può fare?, Raffaello Cortina, Milano 2015.

P. Barile, La Costituzione come norma giuridica, Firenze, Barbera 1951.

S. Boscolo, Che fine ha fatto la progressività dell'Irpef?, in Lavoce.info, 23 marzo 2018 (http://www.lavoce.info/archives/52070/fine-la-progressivita-dellirpef/).
A. Brancasi, Bilancio (equilibrio di), in Enciclopedia del diritto, annali VII, Milano 2014.
C. Buzzacchi, Bilancio e stabilità. Oltre l'equilibrio finanziario, Giuffrè Milano 2015. 
D. Cabras, L'introduzione del principio del c.d. pareggio di bilancio: una regola importante per la stabilizzazione della finanza pubblica, in «Quaderni costituzionali», n. 1, 2012.

L. Carlassare, Priorità costituzionali e controllo sulla destinazione delle risorse, in «Costituzionalismo.it», n. 1, 2013.

L. Carlassare, Diritti di prestazione e vincoli di bilancio, in «Costituzionalismo.it», n. 3, 2015.

L. Carlassare, Solidarietà: un progetto politico, in «Costituzionalismo.it», n. 1, 2016.

I. Ciolli, I diritti sociali, in F. Angelini e M. Benvenuti, Il diritto costituzionale alla prova della crisi economica, Jovene, Napoli 2012.

I. Ciolli, L'art. 81 della Costituzione: da limite esterno al bilanciamento a super principio, in «Forum di Quaderni costituzionali», 26 maggio 2015, (http://www.forumcostituzionale.it/ wordpress/wp-content/uploads/2015/05/ciolli.pdf).

L. Ferrajoli, Diritti fondamentali, Laterza, Roma-Bari 2001.

L. Ferrajoli, Principia iuris. Teoria del diritto e della democrazia, vol. I, Teoria del diritto, Laterza, Roma-Bari 2007.

G. Ferrara, Regressione costituzionale, in Costituzionalismo.it, 18 aprile 2012.

M. Franzini e M. Pianta, Disuguaglianze. Quante sono, come combatterle, Laterza, RomaBari 2016.

S. Gambino (a cura di), Diritti sociali e crisi economica. Problemi e prospettive, Giappichelli, Torino 2015.

M.S. Giannini, L'amministrazione pubblica dello Stato contemporaneo, Cedam, Padova 1988.

T.F. Giupponi, Il principio costituzionale dell'equilibrio di bilancio e la sua attuazione, in «Quaderni costituzionali», n. 1, 2014.

P. Häberle, Le libertà fondamentali nello Stato costituzionale, La Nuova Italia Scientifica, Roma 1993.

H. Kelsen, La democrazia, Bologna 1984 (ed. orig. 1929).

M. Losana, Il riconoscimento del principio di uguaglianza sostanziale nell'ordinamento dell'Unione europea: modelli di riconoscimento, tecniche di realizzazione, strumenti di garanzia, Jovene, Napoli 2010.

M. Luciani, L'equilibrio di bilancio e i princìpi fondamentali: la prospettiva del controllo di costituzionalità, relazione al seminario «Il principio dell'equilibrio di bilancio secondo la riforma costituzionale del 2012», Corte costituzionale, Roma, 22 novembre 2013 (http:// 
www.cortecostituzionale.it/documenti/convegni_seminari/Seminario2013_Luciani.pdf).

M. Luciani, Diritti sociali e livelli essenziali delle prestazioni pubbliche nei sessant'anni della Corte costituzionale, in «Rivista AIC», n. 3, 2016.

D. Messineo, La garanzia del "contenuto essenziale” dei diritti fondamentali. Dalla tutela della dignità umana ai livelli essenziali delle prestazioni, Giappichelli, Torino 2012.

A. Morrone, Il bilanciamento nello stato costituzionale. Teoria e prassi delle tecniche di giudizio nei conflitti tra diritti e interessi costituzionali, Giappichelli, Torino 2014.

A. Morrone, Pareggio di bilancio e Stato costituzionale, in «Rivista AIC», n. 1, 2014.

A. Pace, Il diritto all'istruzione nel tempo di crisi, in «Astrid Rassegna», n. 4, 2013.

C. Pinelli, Interpretazione e interpreti dell'equilibrio di bilancio, in «Astrid Rassegna», n. 5, 2015.

F. Pizzolato, Il minimo vitale. Profili costituzionali e processi attuativi, Giuffrè, Milano 2004.

A. Poggi, Crisi economica e crisi dei diritti sociali nell'Unione Europea, in «Rivista AIC», n. 1, 2017.

G. Razzano, Lo "statuto" costituzionale dei diritti sociali, relazione al Convegno annuale dell'Associazione "Gruppo di Pisa" sul tema I diritti sociali: dal riconoscimento alla garanzia. Il ruolo della giurisprudenza, Trapani, 8-9 giugno 2012 (http://www.gruppodipisa. it/wp-content/uploads/2012/05/trapanirazzano.pdf).

A. Ruggeri, La Consulta rimette abilmente a punto la strategia dei suoi rapporti con la Corte EDU e, indossando la maschera della consonanza, cela il volto di un sostanziale, perdurante dissenso nei riguardi della giurisprudenza convenzionale (" a prima lettura" di Corte cost. $n$. 264 del 2012), in «Consulta online», Sezione "Studi e Commenti”, 2012.

C. Salazar, Crisi economica e diritti fondamentali. Relazione al XXVIII Convegno dell'Aic, in «Rivista AIC», n. 4, 2013.

G. Scaccia, L'equilibrio di bilancio fra Costituzione e vincoli europei, in «Osservatorio sulle fonti», n. 2, 2013.

C. Schmitt, Le categorie del «Politico», Bologna 1972 (ed. orig. 1927).

A. Spadaro, Diritti sociali di fronte alla crisi (necessità di un nuovo "modello sociale" europeo: più sobrio, solidale e sostenibile, in «Rivista AIC», n. 4, 2011.

A. Tabacchi, L'equilibrio dei bilanci: una regola costituzionale "europea" per le finanze pubbliche, in «Rassegna parlamentare», n. 1, 2013.

G. Zagrebelsky, Il diritto mite. Legge diritti giustizia, Einaudi, Torino 1992. 
G. Zagrebelsky, Problemi in ordine ai costi delle sentenze costituzionali, in Aa.Vv., Le sentenze della Corte costituzionale e l'art. 81, u.c. della Costituzione, Atti del seminario svoltosi in Roma, Palazzo della Consulta, nei giorni 8 e 9 novembre 1991, Giuffrè, Milano 1993.

G. Zagrebelsky e V. Marcenò, Giustizia costituzionale, Il Mulino, Bologna 2012.

SOBRE O AUTOR:

\section{Francesco Pallante}

Professor de Direito Constitucional da Universidade de Turim, Itália. Autor de: Il neoistituzionalismo nel pensiero giuridico contemporaneo (Jovene, 2008) e Per scelta o per destino? La Costituzione tra individuo e comunità

(Giappichelli, 2018).

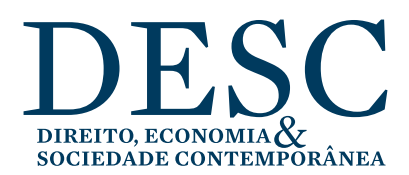

